C A S E

IN WHICH THB

\title{
VENA CAVA INFERIOR
}

\section{WAS OBSTRUCTED FROM THE COMMENCEMENT OF THE COMMON ILIAC VEINS, AND ITS CAVITY ENTIRELY OBLITERATED BETWEEN THE ENTRANCE OF THE EMULGENT AND HEPATIC VEINS.}

Bx THOMAS BEVILL PEACOCK, M.D., Physician to the ROYal General dispensary, aldersgate gtreet.

Communicated by ALEXANDER NASMYTH, Esq.

Received October 28th-Read November 12th, 1844.

Thovgr a considerable number of instances are recorded, in which the main venous trunks of the upper or lower portions of the body have been found more or less completely obstructed, it is conceived that the following case, which furnishes an unusual example of an entire obliteration of the inferior cava, will be interesting to the Society.

Agnes $M^{\prime}$ 'Ewen, aged 47 , of intemperate habits, was admitted into the Royal Infirmary of Edinburgh, on the 16th of August 1842 ; her friends stated that she had been married at the age of 25 ; had had six children, and always recovered well after her confinements. In the October preceding her admission into the Infirmary, her health began to decline, and the catamenia appeared more frequently, and the discharge was more copious than previously. In the VOL. XXVIII. 
course of the spring she received a severe blow on the abdomen, and the following day had hæmorrhage from the bowels. After this her health rapidly gave way, and the catamenia ceased ; the abdomen became swollen, and one month before her admission her legs also were observed to be odematous, the urine being at the same time scanty and high-coloured. Two days before her death she was seized with profuse vomiting of blood, and when received into the Infirmary was greatly prostrated, her countenance was pale, puffy, and sallow, the limbs very œdematous, and the abdomen large and tense. Shortly after being removed to the ward, the hæmatemesis recurred, and bloody fluid was discharged from the bowels. She sank eight hours after her admission.

The autopsy took place thirty-seven hours after death.

The body was very anasarcous, and the abdominal cavity contained ten pints of a pale straw-coloured fluid.

The right lung was adherent to the costal and diaphragmatic pleuræ by old cellular attachments. The left lung was universally adherent, and at the base of the lung the false membrane had undergone the fibro-cartilaginous degeneration: it was full half an inch in thickness, and of a glistening white colour; the lung was of small size, and the corresponding portion of the chest was contracted. Both lungs were œdematous. The heart was natural, weighing $9 \frac{3}{4} \mathrm{oz}$. avoirdupois : its muscular substance was pale. The epithelium of the mucous membrane 
of the œsophagus, near its termination, allowed of being readily removed.

The stomach contained a large quantity of a thin bloody fluid, but scarcely any coagula ; the mucous membrane when washed was of a pale yellowish white colour, except in one or two places where there existed slight redness, which as the membrane was throughout of natural firmness, allowing of being drawn off in streaks of the usual length, was regarded as resulting from imbibition: no ulceration or abrasion was anywhere detected. The small and large intestines were healthy, the glands of Peyer were slightly elevated above the adjacent mucous membrane, and distinctly defined.

The liver was extremely small, weighing only $24 \mathrm{oz}$.; but, with the exception of some opacity of its serous coat, was free from disease; numerous large venous branches ramified on its convex surface.

The spleen was large, weighing $9 \frac{1}{2} \mathrm{oz}$., and moderately firm; several small extravasations of blood were found in its substance.

The left kidney was reduced to a very small size, weighing only $1 \frac{1}{2}$ oz. : it was scabrous and lobulated, of a pale clay colour, and covered with numerous small cysts ; the pelvis was expanded, and the striated portion almost entirely destroyed. The right kidney was large, weighing $5 \frac{1}{2} \mathrm{oz}$. : it was of a pale colour, and on section the granular deposit was found extensively infiltrated into the striated portion, and between the pyramidal bodies.

The uterus was somewhat larger than usual, and 
was attached by firm adhesions to the rectum, sigmoid flexure of the colon, and the sides of the pelvic cavity.

On removing this organ from the body, the veins in its substance, in the broad ligaments, and around the ovaries, were found distended by firm fibrinous clots, and on following the venous branches, in the course of the circulation, the common iliac veins displayed similar coagula, and they thence extended continuously throughout the cava, till, about $1 \frac{1}{2}$ in. below its passage through the diaphragm, that vessel became reduced to an impervious ligamentous cord. The fibrinous clots on the right side, though firmly obstructing the common iliac vein, ceased immediately above its division; but on the left side they extended both into the hypogastric and external iliac veins, entirely closing their cavities. The left common iliac vein was firmly adherent to its corresponding artery.

In the veins of the uterus and ovaries, the coagula had a peculiar fusiform shape, and were firmly adherent to the coats of the vessels, generally by one extremity: they were very solid, so large as to distend the vessels, and generally about half an inch in length, and were composed of lymph in layers of a pale colour, alternating with others of a pinkish hue. In the iliac vessels and cava they consisted of continuous masses, distinctly laminated, not unlike the fibrinous layers of an aneurismal sac. Their colour in the iliacs and lower part of the cava was pale red, and they gradually became more completely decolorized 
as they advanced towards the heart ; they were closely united to the tunics of the veins, and entirely filled their cavities. The spermatic veins were of large size, and their coats thicker and firmer than usual; the orifice of the left vein into the trunk of the emulgent, and of the right into the cava, were closed by firm fibrinous masses, and the course of the right spermatic vein was occupied by small fusiform clots adherent to its coats, and similar to those of the uterine veins.

The calibre of the iliac veins, and especially of the cava, was much reduced, and their coats were indurated and thickened: this became more conspicuous towards the upper part of the vessel;the cavity occupied by the clots gradually diminishing, aud the coats increasing in thickness, till, as before stated, about an inch and a half below the passage of the vessel through the diaphragm, it became entirely obliterated, and was reduced to a mere cord, about the size of the little finger, of a glistening white colour, and firm fibrous texture.

The communication of the left emulgent vein with the cava was open, and this vessel was throughout free from coagula; a vein of considerable size, also unobstructed, entered it from above. The right emulgent vein was closed by a large and closely adherent coagulum, projecting into its orifice.

The heart, with the liver, and other abdominal viscera, having been removed from the body before the obstruction of the cava was detected, I was unable to institute so satisfactory an examination as 
would have been desirable, into the course by which the venous blood of the lower extremities had been returned to the heart. On examination, however, the vena azygos was found of unusually large size, being immediately above the entrance of the vena azygos minor, fully one inch in circumference; the branches entering the right vein, and the vena azygos minor with its branches, and especially some from the spinal canal and intercostal spaces, were greatly enlarged. The portal trunk and branches were free from disease; the hepatic veins entered the only portion of the vena cava inferior which remained pervious.

Though the convex surface of the liver was covered by enlarged veins, the diaphragmatic veins were not materially dilated.

The veins of the integuments of the abdomen, and those following the course of the internal epigastric artery, did not appear larger than usual. Those of the upper extremities and head were free from any appearance of disease. But little blood was contained in the cavities of the heart, or in the vessels. It was everywhere of a very pale colour, and almost entirely fluid.

The above case possesses several points of interest :-

1. It evinces the facility with which the venous circulation can be maintained notwithstanding the entire obstruction of one of its main trunks. In this respect it is not less remarkable than the case related by Dr. Baillie, in which " the inferior cava 
was changed into a ligamentous substance, from the entrance of the emulgent veins to the right auricle of the heart," yet we are assured that this condition was in no degree accessory to the death of the patient. Cases of this description, in which the obliteration of the vessels is complete and of long duration, are decidedly rare, though not a few instances are recorded of partial or recent obstructions in the cavities of the inferior and also of the superior cava. The history of the present case is unfortunately very defective : the swelling of the abdomen, we are informed, was not observed till three or four months before the death of the patient, and the legs became cedematous only one month before that event; these symptoms may therefore fairly be ascribed to the atrophied condition of the liver, and advanced state of disorganization of the kidneys, rather than to the disease of the veins, since the entire obstruction of the upper part of the cava, and its conversion into a firm, white, fibrous cord, must, it is conceived, have required for its completion, a much longer period. The formation of the obstruction in the veins was most probably due to an attack of uterine disease, of which we possess no history, and which probably occurred some time anterior to the symptoms which preceded the fatal event. The absence of any report of such affection will excite little surprise in those familiar with the defective and erroneous statements of hospital patients and their friends.

2. There could be little doubt that the course 
of the venous circulation had been entirely re-established before death, though the exact means by which the blood from the lower extremities was returned to the heart could not be fully traced, from the advanced stage of the examination when the obstruction of the cava was detected. The observations which were made, render it, however, probable that the largest portion found its way into the superior cava through the vena azygos. That vessel was found greatly dilated, being fully double its ordinary size, and the intercostal branches, and those proceeding from the spinal canal, were also much enlarged. The freedom of anastomosis between both divisions of the vena azygos, and the spinal and lumbar veins, would afford a ready channel for the returning column of blood, which may have reached those vessels through the medium of the ileo-lumbar and circumflexa ilii veins. The communication between the left emulgent vein and the commencing branch of the vena azygos minor would allow the return of the blood from that vessel.

Though the branches of the vena porta freely anastomose with the systemic veins, more especially through the medium of the vena mesenterica inferior, and may thus, in cases when the cava is obstructed below the entrance of the hepatic veins, assist in maintaining the circulation, it is not probable that in the present instance this channel was materially concerned in conveying the blood from the lower extremities to the heart. The liver, it will be seen, though free from other morbid change, was very 
much reduced in size, being less than half its usual weight; the portal circulation must therefore have been much impeded, and hence, most probably, the dilatation of the venous branches on the surface of the liver. Mr. Kiernan* has shown that the capsular veins of that organ are branches of the portal system, and anastomose with the phrenic veins, and thus become the medium of a collateral circulation, in some cases of atrophy of the liver. M. Reynaud, $\dagger$ in a case of obstruction of the vena cava and portal trunk, found the veins on the surface of the liver dilated, and traced them to a common trunk which pierced the diaphragm, and opened into the cava above the seat of the obstruction.

There seems no reason to suppose that the integumental veins of the abdomen and thorax, and the epigastric, thoracic, and mammary veins, materially contributed to form collateral channels for the conveyance of the blood.

3 . The larger or smaller venous trunks may become obstructed either as the result of inflammation of the vascular tunics ; by pressure exercised on their coats by aneurismal or other tumours; by the accumulation of morbid matters, fungoid or cancerous, in their cavities ; or, lastly, by the coagulation of the contained blood from retarded circulation through diseased organs, or in peculiar states of system. Instances of obliterations of the cava traceable to

* The Anatomy and Physiology of the Liver. Philosophical Transactions, 1833, p. 733.

† Journal Hebdomadaire de Médecine, tom. v. p. 173. 
these several causes are referred to in the Appendix. In the case narrated in the paper, the disease was evidently inflammatory in its origin, though from the defective history of the patient's previous state of health it is not easy to assign the period at which the inflammation had occurred. From the same cause it is impossible to ascertain the relation which the disease of the veins bore to the affection of the kidneys, which was the immediate cause of death. The frequency of affections of the endocardium lining the cavities and covering the valves of the heart, and of the inner coat of the aorta, in persons who have died from the effects of granular degeneration of the kidneys, has been dwelt upon by all writers on that disease:- the similar tendency to secondary affections of the venous system appears however to have attracted little attention. In 1835 Dr. Osborne* described the emulgent veins as occasionally found obstructed by coagula in cases of granular degeneration, and the same observation has since been repeated by Rayer, $\dagger$ and Stokes, $\ddagger$ and more recently by Dr. Chevers, $\|$ who also refers to the not unfrequent occurrence of phlebitis of the veins of the extremities in persons labouring under renal disease. I have three or four times found firm, partially decolorized, and more or less adhe-

* The Nature and Treatment of Dropsical Diseases, $12 \mathrm{mo}$. Dublin, 1835.

† Maladies des Reins, tom. ii. p. 104. Paris, 1840.

† Dublin Journal, vol. xxi. p. 144. 1842 .

|| Guy's Hospital Reports, vol. viii. p. 95. 
rent coagula, in the veins of diseased kidneys; and have twice observed phlebitis of the femoral and inguinal veins occurring during the course of the chronic form of disease, and after death have found those veins obliterated by fibrinous exudations adherent to their parietes. It seems, therefore, that phlebitis is by no means an uncommon complication of renal disease ;* and it may be inferred that the lining membrane of the vascular system generally, like the serous membranes to which it bears so close an analogy, is peculiarly liable to inflammatory action in persons labouring under that affection.

4. The examination of the mucous membrane of the stomach and intestines afforded no explanation of the source of the hæmorrhage which occasioned the death of the patient;-no ulceration, abrasion, or even congestion being anywhere de-. tected. The absence of the signs of disease of the mucous membranes where intestinal affections have been predominant during life, appears to be not unfrequent in the bodies of persons who have died of disease of the kidneys. I have found the mucous membrane pale, of natural firmness, and free from any appearance of disease, throughout the whole canal, in patients who were mainly carried off by the secondary diarrhœa and dysentery.

* Dr. Bright, in the first volume of his Medical Reports, has related the case of a young woman who died of renal dropsy, and in whom the portal vein and its hepatic branches, with the splenic vein and the pulmonary artery, were filled with firm clots, partially deprived of red particles. Case 6, p. 19. 


\section{A P P E N D I X,}

CONTAINING REFERENCES TO THE CASES OF OBSTRUCTION OF THE SUPERIOR AND INFERIOR CAVA, PREVIOUSLY PUBLISHED.

1. Obstruction resulting from inflammation of the coats of the veins.

INFERIOR CAVA.

By far the larger number of cases of obstruction of the inferior cava are of this description, and specimens exhibiting the disease are contained in several of the Museums in London. In all the preparations, however, which I have had an opportunity of examining, and probably in nearly all the published cases, the change was recent, and the cavity of the vein was obstructed by fibrinous masses. The case recorded by Dr. Baillie, and that described in the paper, afford, so far as I am aware, the only examples in which the trunk of the inferior cava was converted into an impervious cord. Probably in most cases, as in that related, the disease has commenced in the uterine veins, and been thence extended into the cava.

Baillie,-Transactions of a Society for Improvement in Medical and Chirurgical Knowledge, vol. i. p. 127. - The vein converted into a ligamentous cord, from the entrance of the emulgent to the hepatic veins. Collateral circulation assisted by two veins following the course of the vena azygos. 
Wilson, in ibid. vol. iii. page 65.-Recent obstruction of the vein the sequel of puerperal phlebitis.

Haller,-Pathological Observations, London 1756 , p. 41, and Philosophical Transactions, vol. xliv. $1746 \& 47$, p. 527 . - Vein closed by a fibrinous mass from the emulgent veins to its division into the iliacs. Collateral circulation by the right spermatic vein.

Reynaud,-Journal Hebdomadaire de Médecine, tom. v. p. 173.-Obstruction extending for three inches below the auricle, and involving also the hepatic veins and the right branch of the vena porta. The coats of the veins thickened, and fibrinous deposit on their exterior. Collateral circulation through the external abdominal veins.

Journal Universel et Hebdomadaire, tom. ii. p. 381.-Fibrinous clots extending from the entrance of the emulgent veins to the iliac and femoral veins, but not producing complete obstruction. The vena porta contracted and containing coagula.

Laennec,-Archives Générales de Médecine, tom. vi. p. 619.-A preparation of the inferior cava and right iliac vein obstructed by clots exhibited to the Académie de Médecine de Paris, at the desire of M. Laennec.

Knape,-Hufelands Journal der Practischen Arzneikunde und Wundarzneikunde, vol. xxxvi. p. 12. The vein closed by the adhesion of its coats.

Gross,-Pathological Anatomy, Philadelphia, vol. i. p. 295, notice in Dublin Journal of Medical Science.-Vein closed by a plug of lymph from the 
second lumbar vertebra to the liver. Clots in the portal vein, and some of the veins of the left lung.

Bright,-Medical Reports, vol. ii. p. 66, Case xxiv.-Coagulum, yellow and laminated, and in some places presenting an appearance of suppuration, occupying the cava and iliacs from beneath the liver to Poupart's ligament.

Carswell,-Fasciculus of Plates illustrating the formation of Analogous Tissues, plate 2nd, fig. 5.Lower portions of the cava and the iliac veins obstructed by adherent fibrinous masses.

Rayer,-Maladies des Reins, plate 50, fig. 4.-The cava and emulgent veins obstructed by clots, the sequel of puerperal phlebitis.

\section{SUPERIOR CAVA.}

Complete obstructions of the superior vena cava are extremely rare : of the nine cases to which I am able to refer, three only appear to have been complete. The most frequent cause of obstruction in this vessel is the pressure of aneurismal or other tumours. The following cases may probably be the result of inflammation :-

Lobstein,-Anatomie Pathologique, t. ii. p. 110.The vein closed by a solid mass, which by its pressure had caused obstruction of the two right pulmonary veins,-quoted from Weissbrod's Observat. Anat. Duæ.

J. Reid,-Edinburgh Medical and Surgical Journal, vol. xliii. p. 297. - The vessel converted into a fibrous cord. Cause of obstruction not ascertained, 
but it is suggested to have been possibly owing to the pressure of diseased bronchial glands.

Breschet,-French Translation of Hodgson on Diseases of the Arteries, \&c., t. ii. p. 480 , note.A model contained in the Musée de la Faculté de Médecine de Paris, of an obstruction of the cavity of the superior cava by a fibrinous mass, prepared under the direction of M. Dupuytren.

2. Obstructions resulting from pressure on the trunks of the veins.

INFERIOR CAVA.

Cline,-Wishart's Trans. of Scarpa on Aneurism, note, p. 20.-The vein obliterated shortly above its bifurcation, by a tumour originating behind the peritoneum.

Bouillaud,-Archives Générales de Médecine, tom. vi. p. 567. - The lower portion of the vessel obstructed by the pressure of diseased mesenteric and lumbar glands.

Andral,-Clinique Médicale, Diseases of the Liver. - Partial obstruction of the inferior cava and porta by the compression of diseased lymphatic glands.

\section{SUPERIOR CAVA.}

Reynaud,-Journal Hebdomadaire de Médecine, tom. ii. p. 110.-Imperfect obstruction from the pressure of an aneurism.

Dr. W. Hunter,-Medical Observations and Enquiries, vol. i. p. 333.-Similar case. 
Also Otto,-Neue Seltene Beobachtungen, Berlin 1824, part i. p. 65,

And Deckart, in Otto's Pathological Anatomy, by South.

Dr. Wilson, in Report of Proceedings of London Med. and Chir. Soc., London Med. Gazette, vol. xxi. p. 666 (vol.i. for $1837 \& 38$ ). -Two cases of imperfect obstruction of the superior cava from pressure : one of these, in which the compression was produced by an aneurism, is more fully described by $\mathrm{Mr}$. Shaw, Med. Gaz., vol. xxvi. p. 522 (vol. ii. for 1839 \& 40). Dr. Watson alludes to a case of this kind in his lectures, probably that of Dr. Wilson. I have seen the left vena innominata and the trunk of the vena azygos entirely obliterated from the pressure of aneurisms, and the right iliac and inguinal veins filled with fibrinous clots from the compression of the vein by an uterine tumour.

3. Accumulation of morbid deposits in the cavity of the veins.

CAVA INFERIOR.

Cruveilhier,-Anatomie Pathologique, Livraison 5, p. 3 \& 4.-Medullary matter filling the vena cava as far as the kidney, in a person from whom the testis had been removed; - a band of fungoid glands found along the lumbar portion of the spine.

Rayer,-Maladies des Reins, plates $47 \& 49$.Vena cava inferior and emulgent vein, filled with pale 
deposit in a case of fungoid disease of the kidney ; the deposit in the veins was probably of a similar nature to the disease of the kidneys.

Barlow,-Guy's Hospital Reports, October 1844.Vena cava inferior distended with medullary matter, and its lower extremity contracted in connection with fungoid disease of the kidney. Fungoid matter is probably only found in the veins, when the same disease affects adjacent organs, and chiefly in the smaller veins of the organs so diseased. Cruveilhier, in Livraison xviii. plate 1, has figured an obliteration of the hepatic vein by medullary deposition; and Carswell has given drawings of the same in the portal and renal veins, and in the veins of the stomach. I have seen the trunk of the vena porta and its branches in the liver filled with a pale deposit, probably carcinomatous, in a case of medullary sarcoma of the liver.

4. Obstruction of the veins by coagula resulting from stasis of the contained blood.

This cause of venous obstruction is scarcely likely to o perate in the main venous trunks. The only examples to which I can refer are the clots found in the veins of the liver and kidneys, in some forms of chronic disease of those organs in which the coats of the veins present no appearances of inflammation. The obstructions in the veins of the extremities occurring in persons exhausted by long continued diseased action, or great loss of blood, may probably be 
in part ascribed to the enfeebled circulation combined with a peculiar condition of the blood. In most of these cases, however, a certain degree of excited action in the affected vessel appears to attend the formation of the coagula; though the number of vessels consentaneously affected show how much this excited action is connected with general causes. See cases detailed by Dr. Bright in his Medical Reports, vols. i. \& ii.; and also the cases of Reynaud and Gross.

Lobstein has described a case in which he found the veins of a limb long paralysed, contracted, and containing clots, which he ascribed to the coagulation of the blood from loss of nervous energy. He says, " Cette derniére cause m'a paru évidente dans un cas d'hemiplégie qui s'est terminé par la mort. Les parois du veines du côté paralytique étaient toutes plus ou moins obstruées par des polypes, tandis que celles du côté sain renfermaient du sang dissous et fluide."-Traité d'Anatomie Pathologique, tom. ii. p. 610 .

In the Compendium of Pathological Anatomy by Otto, there are references to one or two cases which I have not been able to find: he also refers to the following :-

Rhodius,-Mantissa Anatomica, obs. xxi. p. 15.The trunk of the vena cava so completely obstructed that it would not allow the passage of a probe : " haud dubie ab exulceratione a vermibus in ramo iliaco repertis excitata."

Bartholinus (Th), - Hist. Anat. Rar., cent. ii. hist. 35. - The vena cava obstructed near the heart. 
Bontius,-De Medicinâ Indorum, fol. 37.-The vena cava closed by a fatty substance.

Albinus,-Annotationes Academicæ, lib. 7, c. 9. -The vena cava obliterated near the origin of the iliacs.

In a note to Portal's Anatomie Médicale, t. iii. p. 437, Chopart is stated to have seen in England an obliteration of the inferior vena cava, in which the circulation was maintained by means of the epigastric and internal mammary veins.

A fuller account of several of the cases here referred to is given by Dr. W. Thomson, in vol. xliii. of Edinburgh Medical and Surgical Journal. See also Rokitansky, Handbuch der Pathologischen Anatomie, ii Band. p. 674. 